\title{
Eficiencia reproductiva de búfalas multíparas sometidas a implantes de melatonina fuera de la estación reproductiva
}

\author{
Sousa, A.O. ${ }^{1}$; Baruselli, P.S. ${ }^{2}$ Carvalho, N.A. ${ }^{2}$; Konrad, J.L. ${ }^{3}$; Crudeli, G.A. ${ }^{3}$ \\ ${ }^{1}$ Departamento das Clínicas Veterinárias, UEMA, São Luis, Brasil. ${ }^{2}$ Departamen-to de Reprodução Animal USP, \\ São Paulo, Brasil. ${ }^{3}$ Cátedra de Teriogenología, Facultad de Ciencias Veterinarias, Universidad Nacional \\ del Nordeste, Sargento Cabral 2139, Corrientes (3400), Argentina. E-mail: gacrudeli@hotmail.com.
}

\begin{abstract}
Resumen
Sousa, A.O.; Baruselli, P.S.; Carvalho, N.A.; Konrad, J.L.; Crudeli, G.A.: Eficiencia reproductiva de búfalas multíparas sometidas a implantes de melatonina fuera de la estación reproductiva. Rev. vet. 23: 1, 38-42, 2012. El objetivo de este trabajo fue evaluar el efecto de implantes subcutáneos conteniendo $18 \mathrm{mg}$ de melatonina (Melovine ${ }^{\circledR}$ ) sobre la ciclicidad de las búfalas durante el fotoperíodo largo. La eficacia de los tratamientos fue evaluada a través de la aparición del estro, utilizando reproductores con bozal marcador. Se utilizaron 100 búfalas distribuídas en tres grupos: G1 (grupo control, $n=38$ ), G2 (grupo con un implante, $n=36$ ) y G3 (grupo con tres implantes, $\mathrm{n}=26$ ). Ochenta y seis búfalas manifestaron actividad cíclica en el fotoperíodo largo y 71 de ellas se preñaron $(82,6 \%)$. Catorce hembras permanecieron en anestro durante todo el período experimental, 6 pertenecientes al G1 (15,8\%), 6 al G2 (16,7\%) y 2 al G3 (7,7\%). De las 38 búfalas del G1, $32(84,2 \%)$ registraron celo y fueron servidas; de ellas, $26(68,4 \%)$ se preñaron en noviembre y las 6 restantes $(15,8 \%)$ en marzo. Del G2, 30 búfalas $(83,3 \%)$ fueron servidas y 23 de ellas $(63,9 \%)$ quedaron preñadas en noviembre y siete $(19,4 \%)$ en marzo. En G3 fueron servidas 24 (92,3\%), de las cuales $22(84,6 \%)$ se preñaron en noviembre y dos $(7,7 \%)$ en marzo. Las tasas de celo y preñez no registraron diferencias significativas entre grupos con o sin implantes de melatonina ( $p>0,05$ ). Se concluye que el $86 \%$ de las búfalas puede presentar actividad reproductiva hasta un fotoperíodo con luminosidad de aproximadamente 13 horas, no siendo influenciadas por la administración de melatonina.
\end{abstract}

Palabras clave: búfalo, reproducción, fotoperíodo, implante de melatonina, preñez.

\begin{abstract}
Sousa, A.O.; Baruselli, P.S.; Carvalho, N.A.; Konrad, J.L.; Crudeli, G.A.: Reproductive efficiency of multiparous buffaloes subjected to implants of melatonin out of breeding season. Rev. vet. 23: 1, 38-42, 2012. The objective of this work was to evaluate the effect of melatonin implants on reproductive function of buffaloes during spring and summer (long photoperiod). The efficiency of treatments was tested evaluating oestrus behavior, using teaser bulls with reproductive device markers. The experiment included 100 females distributed in three groups: G1 (control group, n=38), G2 (one melatonin implant, $\mathrm{n}=36$ ) and G3 (three implants, $\mathrm{n}=26)$. Eighty six ( $86 \%$ ) females were in cyclical activities in the long photoperiod. They were mated and $71(82.6 \%)$ became pregnant. It was verified that 18 animals repeated heat totalizing 21 repetitions. Fourteen females remained in anestrous during all the experiment, being 6 of group G1 (15.8\%), 6 of group G2 (16.7\%) and 2 of group G3 (7.7\%). From 38 females of G1, 32 (84.2\%) showed oestrus and were mated, being 26 (68.4\%) pregnant in November and $6(15.8 \%)$ in March. In G2 $(\mathrm{n}=36) 83.3 \%(\mathrm{n}=30)$ were observed in oestrus, being 63.9\% $(\mathrm{n}=23)$ pregnant in November and 19.4\% (7) in March. In G3 animals $(\mathrm{n}=26) 92.3 \%$ $(\mathrm{n}=24)$ showed oestrus, being $84.6 \%(\mathrm{n}=22)$ pregnant in November, and $7.7 \%$ (2) in March. The results showed reproductive activity in $86 \%$ of female buffaloes, with no differences $(p>0.05)$ between groups, indicating that reproductive behaviour of this species prolongs almost until the end of the year. Consequently, reproductive behaviour was observed during the long photoperiod months, indicating that in Brazil the pattern of reproductive behaviour is different for this species. Moreover, the administration of melatonin implants did not affect reproductive pattern during this period.
\end{abstract}

Key words: buffalo, reproduction, photoperiod, melatonin implant, pregnancy. 


\section{INTRODUCCIÓN}

La importancia creciente de la bubalinocultura requiere una mayor utilización de la biotecnología disponible. A pesar de que el rodeo brasilero tiene una población estimada de cuatro millones de cabezas de búfalos, fruto del crecimiento anual del $13 \%{ }^{3}$, la mayoría no ha mejorado su potencial genético, principalmente por localizarse en una región de difícil acceso, como lo es el Valle del Amazonas ${ }^{26}$, dentro del cual la Isla de Marajo se destaca como el principal centro productivo ${ }^{21}$, donde diferentes razas y subespecies se encuentran cruzadas totalmente al azar ${ }^{26}$.

Debido al hecho de que los búfalos karabao (Bubalus bubalis limneticus) poseen solo 48 cromosomas $\mathrm{y}$ los búfalos de río (Bubalus bubalis fluvialitis) poseen 50 , sus cruzamientos están provocando problemas de orden cromosómico consecuentes a dicha hibridación ${ }^{21,26}$. Los rodeos bubalinos que se destacan por su productividad están localizados principalmente en el sur, sudeste y nordeste de Brasil y a pesar de ser poco numerosos exhiben un mayor potencial productivo, a diferencia del numeroso rodeo existente en Marajó y en el valle amazónico.

La aptitud reproductiva durante el fotoperíodo corto es muy elevada en el sur y sudeste de la zona, lo que configura un patrón productivo con producción fuera de época de demanda de carne y leche, reflejándose este hecho en la subutilización de las industrias lácteas especializadas ${ }^{4}$. Provoca además problemas de eficiencia reproductiva, que van desde el retraso de la pubertad hasta la falta de expresión del celo y ovulación ${ }^{22}$.

La glándula pineal produce melatonina, hormona que determina el establecimiento del carácter reproductivo estacional ${ }^{12}$. Los perfiles de melatonina y su variación dinámica a lo largo del año hacen de la pineal la responsable de la regulación de los procesos fisiológicos necesarios para la adaptación de los animales a la variabilidad ambiental a que están sujetos ${ }^{11} \mathrm{y}$ otros efectos relacionados a la reproducción $6,8,15,17,19,20$. La baja o alta concentración de los niveles plasmáticos de la hormona constituyen señales al medio interno sobre la duración del día y de la noche, las cuales también operan en el reconocimiento de las estaciones del año 10, 24 . Así, las oscilaciones de la luz ambiental adquieren importancia en la regulación de las acciones fisiológicas ligadas a los niveles circulantes de melatonina ${ }^{14}$ 16.

Se considera que el efecto "día-largo" (luminosidad superior a $12,5 \mathrm{~h}$ en $24 \mathrm{~h}$ ${ }^{23}$ ) induce anestro en especies con aptitud para el fotoperiodo corto ${ }^{11}$. El efecto "día-corto" fue obtenido mediante implantes de melatonina ${ }^{11}$, por infusiones de bolos intraruminales ${ }^{19} \mathrm{y}$ también por variahoras). vs horas). ciones de la luminosidad en cámaras climatizadas ${ }^{2}$. En el venado silvestre, cuya aptitud reproductiva ocurre durante los días cortos, la estacionalidad provoca que las glándulas reproductivas permanezcan inactivas durante el fotoperíodo largo, tal como ocurre en caprinos, ovinos y bubalinos 1, 5, 13,22, 28 .

El patrón reproductivo de los bubalinos se caracteriza por evidenciar un reinicio de las actividades cíclicas al comienzo del otoño ${ }^{1,4,22,25}$. De esta forma, muestran actividades reproductivas durante el otoño e invierno, disminuyéndolas en primavera y verano ${ }^{1,}$ 5,22 . Algunas hembras poseen actividad reproductiva ininterrumpida durante el año entero, mostrando un patrón fisiológico de síntesis de melatonina semejante al de los mamíferos poliéstricos ${ }^{18,28}$. En las Figuras 1 y 2 se muestran dos perfiles circadianos durante el equinoccio, para animales estacionales y no estacionales respectivamente ${ }^{18}$.

Teniendo como base estas consideraciones, el presente trabajo tuvo como objetivo estudiar la eficiencia reproductiva de 100 búfalas distribuidas en tres grupos de tratamientos en base a implantes de melatonina durante el período de anestro estacional, a efectos de ampliar conocimientos sobre la reproducción de esta especie, verificar la funcionalidad de los implantes de melatonina en la fertilidad durante el fotoperíodo largo, evaluar la tasa de servicio y de preñez en primavera y verano (octubre a marzo), y aportar conocimientos sobre la desestacionalización en la especie bubalina.

\section{MATERIAL Y MÉTODOS}

El presente trabajo fue realizado en cuatro establecimientos situados en el Valle de Ribeira, Estado de São Paulo (Brasil), localizados entre $24^{\circ} 29^{\prime}$ y $24^{\circ} 42^{\prime}$

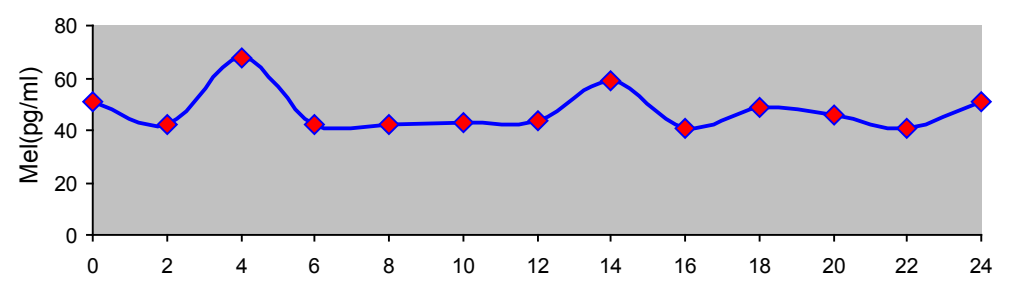

Figura 1. Perfil circadiano de los niveles circulantes de melatonina en animales estacionales durante el equinoccio (melatonina/Mel vs

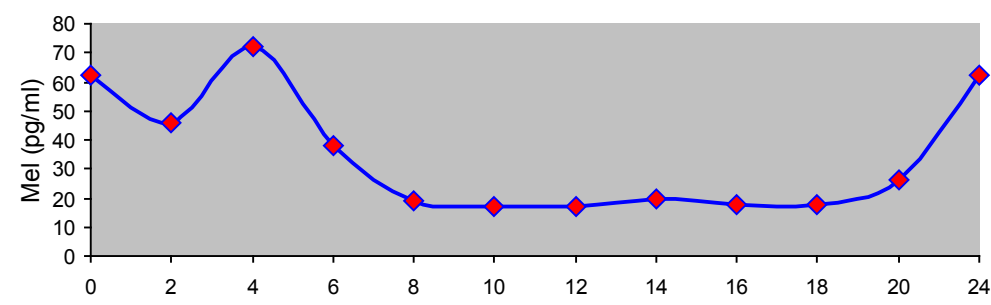

Figura 2. Perfil circadiano de los niveles circulantes de melatonina en animales no estacionales durante el equinoccio (melatonina/Mel 
de latitud sur, en el período de marzo de 2006 a diciembre de 2007. Los animales estuvieron sometidos a un manejo semiintensivo, siendo las búfalas de raza Murrah en su mayoría, las que habían parido en forma normal y no presentaron problemas puerperales. Los toros se retiraron en marzo (inicio del otoño), para mantenerlas vacías hasta la colocación de los implantes, los que fueron aplicados el día 6 de octubre (primavera), junto con la incorporación de los toros al rodeo.

Los animales tuvieron acceso permanente a pasturas, heno picado, sal mineral y agua ad libitum. Fueron formados tres grupos experimentales, siendo: G1 $(n=38)$ control; G2 $(n=36)$ aplicación de un implante y G3 $(n=26)$ aplicación de tres implantes subcutáneos. Cada implante contenía $18 \mathrm{mg}$ de melatonina (Melovine, Ceva Sante Animale ${ }^{\circledR}$ ). Para detectar el celo las búfalas se observaron dos veces al día, por la mañana $(6: 00$ a 7:00 h) y por la tarde $(18: 00$ a 19:00 h), teniendo en cuenta la aceptación al macho y monta completa, considerándose a esta última, como signo de estro. Tales actividades fueron llevadas a cabo durante todo el período experimental.

Los reproductores llevaban colocado un bozal marcador (chin-ball), con pintura de color claro para que su visualización facilitara la detección del celo. La palpación rectal, fue realizada a partir de los 60 días del inicio del trabajo y luego cada 30 días, para la evaluación y/o confirmación del diagnóstico de gestación. La duración del fotoperiodo fue medida semanalmente, iniciándose el 23 de setiembre y finalizando el día 29 de marzo (inicio del otoño).

Los resultados se procesaron a través del análisis de variancia (ANOVA), para evaluar el efecto de los tratamientos sobre las tasas de servicio y preñez (variables binominales). Las diferencias entre las medias fueron evaluadas por el test-t de Student ${ }^{27}$.

\section{RESULTADOS Y DISCUSIÓN}

Los resultados obtenidos se muestran en la Tabla 1. Se encontró que un $14 \%$ de las búfalas permaneció en anestro hasta el final del experimento. El 86\% restante, presentó celos en dos diferentes patrones: un $82,6 \%$ (71/86) fueron servidas hasta la mitad de noviembre y de éstas, un 68,6\% (59/86) quedaron preñadas. En la segunda mitad de noviembre y hasta marzo, solo un $17,4 \%(15 / 86)$ búfalas manifestaron celo y fueron servidas, habiéndose preñado el 13,9\% (12/86).

El porcentaje de distribución del servicio mostró que en G1, un $84,2 \%$ de las hembras (32/38) fueron servidas, siendo que el $68,4 \%(26 / 38)$, lo hicieron hasta noviembre, las restantes del G1, un $15,8 \%(6 / 38)$ lo hicieron desde mediados de noviembre y hasta marzo. En las búfalas correspondiente al G2, del total de 36, fueron servidas un $83,3 \%(30 / 36)$, de las cuales un $63,9 \%(23 / 36)$ y un $19,4 \%(7 / 36)$, fueron servidas hasta noviembre y de mediados de noviembre a marzo respectivamente. En cuanto al G3, del total de búfalas $(\mathrm{n}=26)$, un $92,3 \%(24 / 26)$ fueron servidas, siendo un $84,6 \%(22 / 26)$ hasta noviembre y un 7,7\% (2/26) de diciembre a marzo.

La tasas de servicios fueron de $84,2 \%$ (32/38), $83,3 \%(30 / 36)$ y $92,3 \%(24 / 26)$, para G1, G2 y G3 respectivamente. En cuanto al porcentaje de preñez, los valores fueron de 68,4 (26/38), 63,9\% (23/36) y 84,6\% (22/26) para G1, G2 y G3 respectivamente. No se observaron diferencias estadísticamente significativas $(p>0,05)$ entre tasas de servicio y preñez de los grupos experimentales con o sin implantes. Las observaciones del celo permitieron detectar una intensa actividad cíclica en los dos primeros meses del experimento, con manifestación de la ciclicidad y mayor fertilidad en octubre e inicio de noviembre. La tasa de servicios fue muy baja desde diciembre hasta marzo, en parte porque la mayoría de las búfalas estaban preñadas, y en las restantes se había instalado el anestro estacional fotoperiódico.

En el mes de octubre se detectó una cantidad de 62 servicios, con 37 preñeces, en tanto en el mes de noviembre fueron servidas unas 35 búfalas, con 24 preñeces y en diciembre solo se observaron 8 servicios, de los cuales 5 repitieron celo y luego se preñaron. En el mes de enero, 2 búfalas fueron montadas y preñadas. A su vez, en febrero, hubo solo una búfala servida (que gestó) y dos en marzo, quedando ambas preñadas. Según los servicios totales realizados, se comprobó que 86 fueron servidas del 6 de octubre al 13 de marzo. La estacionalidad se reveló muy rígida en solo 14 búfalas, desde el inicio del trabajo hasta el 6 de octubre, cuando el fotoperíodo inhibió sus actividades reproductivas. Entretanto, hubo 15 hembras, que iniciaron la ciclicidad a partir de la mitad de noviembre, cuando el período de luminosidad superaba las 13 horas.

El efecto del fotoperíodo largo sobre los búfalos es considerado como un inductor del anestro 1, 5, 13, 22, 28 . Varios autores estudiaron la eficiencia reproductiva de búfalas con implantes de melatonina, considerando la hipótesis que estos implantes mantendrían elevada la concentración de melatonina como ocurrió con caprinos ${ }^{7}$ y ovinos ${ }^{11}$, durante las estaciones del fotoperíodo largo. Nuestros resultados indican que el $86,0 \%(n=86)$ de las búfalas fueron servidas durante dos meses de primavera en que el fotoperíodo es largo, siendo estos hallazgos, no concordantes con los resultados de otros trabajos 1, 5, 18, 22, 28. También, se observó que un 14,0\% $(n=14)$ de las búfalas fueron estrictamente estacionales, 
en concordancia a lo mencionado por otros autores ${ }^{18 \text {, }}$ 22, 28 .

Las actividades reproductivas del 86,0\% $(n=86)$ de las hembras durante el período experimental siguieron dos patrones, en el primero de ellos el 71,0\% $(n=71)$ de las búfalas fueron servidas hasta mediados de noviembre, y en el segundo hubo un $15,0 \%(n=15)$ que ciclaron durante el fotoperíodo más largo, concordando con otras observaciones ${ }^{18,28}$. De lo antes explicado, hubo un tercer patrón, que correspondió a aquellas búfalas $14 \%(n=14)$ que fueron absolutamente estacionales, lo que implica que en este experimento hubo tres patrones de comportamiento reproductivo durante el período estudiado.

Las expresiones reproductivas en los tres grupos experimentales no mostraron variaciones estadísticamente significativas $(\mathrm{p}<0,05)$. Estos datos difieren de un reciente trabajo realizado en bubillas en la India, donde se utilizó un implante similar cada $50 \mathrm{~kg}$ de peso vivo, logrando en los animales implantados una ciclicidad del $100 \%$, en tanto que el grupo control no registró actividad ovárica alguna ${ }^{9}$. En el presente ensayo la ausencia de diferencias quizás pueda deberse a una baja dosis de melatonina, pues en el último artículo citado, en las bubillas de $450 \mathrm{~kg}$ se usaron 9 implantes (162 mg), dosis que fue efectiva para lograr la ciclicidad fuera de la estación reproductiva ${ }^{9}$, en tanto que en el presente trabajo, con búfalas adultas y de mayor peso, solo colocamos entre 18 y $54 \mathrm{mg}$ de melatonina.

Un $92,3 \%$ de las búfalas G3 fueron cíclicas en el fotoperíodo largo y $84,6 \%$ fueron servidas hasta noviembre, siendo este hecho discordante con lo acontecido en otro ensayo ${ }^{28}$. Por otra parte, un $7,7 \%$ de las búfalas fueron servidas en el fotoperíodo largo, en coincidencia con otras investigaciones ${ }^{28}$. Los patrones de comportamiento reproductivo aquí hallados en los tres grupos experimentales, fueron diferentes a los mencionados en un reporte anterior ${ }^{7}$.

En conclusión, surge que en el $86 \%$ de las búfalas el fotoperiodo largo no afectó la ciclicidad y en el $71 \%$ no interfirió en la fertilidad hasta la mitad de noviembre. Los implantes de melatonina, no se mostraron eficaces para promover modificaciones en la ciclicidad, quizas debido a la baja dosificación o a que en la región, la variación circadiana es mínima y la estacionalidad reproductiva no es tan rígida, dependiendo más del estado nutricional de las hembras. La fertilidad, expresada en tasas de servicio y de preñez obtenidas entre los grupos experimentales, fue considerada aceptable y no acusó diferencias estadísticamente significativas. Esta investigación debería profundizarse utilizando mayores dosis de melatonina y efectuando seguimiento ecográfico de la onda folicular, a efectos de poder arribar a resultados más certeros.

Agradecimientos. Al Dr. Malpaux y a Ceva Santé Animale, por la provisión de los implantes de melatonina utilizados en el experimento (Melovine $\left.{ }^{\circledR}\right)$. A los Dres. Leónidas Chow Castillo y Otavio Ohashi por su permanente apoyo y colaboración. A la Asociación de Criadores de Búfalos do Vale do Ribeira-Sao Pablo, por proveer los animales y el personal de campo que colaboró en la realización de las tareas.

\section{REFERENCIAS}

1. Abhi HL, Nagpal MP, Sharma TL, Grewal JA. 1973. A study on the breeding behaviour of Murrah buffaloes and their breeding efficiency through artificial insemination under farm conditions. Indian J Dairy Sci 26: 107-113.

2. Arendt TJ. 1998. Melatonin and the pineal gland: influence on mammalian seasonal and circadian physiology. Rev Reprod 3: 13-22.

3. Baeta LN. 1977. Rebanho bubalino atual e suas perspectivas na pecuária brasileira. Publ Ass Bras Criad Búf, Sao Paulo (Brasil), 9 p.

4. Baruselli PS, Mucciolo RG, Visintin JA, Viana WJ, Arruda RP, Madureira EH, Oliveira CA, Molero-Filho JR. 1997. Ovarian follicular dynamics during the estrous cycle in buffalo. Theriogenology 47: 1531-1547.

5. Basu S. 1962. Seasonal variation of fertility in Murrah buffaloes. Indian Vet J 39: 433-438.

6. Cardinali DP. 1981. Melatonin. A mammalian pineal hormone. Endocr Rev 2: 327-346.

7. Chamineau P. 1993. Environment and animal reproduction. World Anim Rev 77: 2-14.

8. Ebbeling FJ, Foster DL. 1989. Pineal melatonin rhythms and the timing of puberty in mammals. Experientia 45 : 946-954.

9. Ghuman SP, Singh J, Honparkhe M, Dadarwal D, Dhaliwal GS, Jain AK. 2010. Induction of ovulation of ovulatory size non-ovulatory follicles and initiation of ovarian cyclicity in summer anoestrous buffalo heifers using melatonin implants. Reprod Domest Anim 45: 600-607.

10. Hastings MH, Vanance G, Mayaywood E. 1989. Some reflections on the phylogeny and function of the pineal. Experientia 45: 903-909.

11. Kennaway DJ, Gilmore TA. 1985. Effects of melatonin implants in ram lambs. J Reprod Fert 73: 85-91.

12. Lerner AB, Case JD, Takahashi Y. 1959. Isolation of melatonin, the pineal gland factor that lightens melanocytes. J Am Chem Soc 80: 25-87.

13. Lincoln GA, Short RV. 1980. Seasonal breeding: Nature's contraceptive. Recent Progr Horm Res 36: 1-32.

14. Loudon A. 1985. A gland for all seasons. New Scientist 25: 40-43.

15. Martin J, Engle J, Klein DC. 1977. Inhibition of the in vitro pituitary response to luteinizing hormone-releasing hormone by melatonin, serotonin and 5-methoxytryptamine. Endocrinology 100: 675-678.

16. Meefford IN, Chang P, Klein DC, Namboodiri MA, Sugden D, Barchas J. 1983. Reciprocal day/night relationship between serotonin oxidation and $\mathrm{N}$-acetilation products in the rat pineal gland. Endocrinology 113: 15821586.

17. Nakazawa K, Marubayashi U, McCann SM. 1991. Mediation of the short-loop feedback of luteinizing hormone (LH) on LH-releasing hormone release by melatonin-in- 
duced on inhibition of LH release from the pars tuberalis. Proc Nat Sci USA 88: 7576-7579.

18. Parmeggiani A, Di Palo R, Ziccarelli L. 1994. Melatonina e stagionalitá riprodutiva della buffalla. Agric Ric 153: 41-48.

19. Poulton AL, Brown DC, Thomas EM, Kelly MI, Symows AM, Arendt J. 1988. Use of an intraruminal soluble glass bolus containing melatonin for early lamb production. The Vet Rec 122: 226-228.

20. Reiter JR. 1996. Mechanisms of control of reproductive physiology by the pineal gland and its hormones. Tese Doutorado, Escola de Veterinária, Universidade Federal de Minas Gerais, Brasil, 125 p.

21. Ribeiro HF. 1996. Puerpério na búfala: Aspectos clínicos e histológicos da involução uterina e etividade ovariana. Tese Doutorado, Escola de Veterinária, Universidade Federal de Minas Gerais, Brasil 125 p.

22. Sousa AO, Baruselli PS, Ohashi OM, Oliveira CA, Solano FR, Blume H, Santos HP. 1999. Puberdade em fêmeas murrah (Bubalus bubalis) do Vale do Ribeira-SP. Rev Bras Reprod Anim 23: 173-175.
23. Stanisiewki EP, Ames NK, Chapin LT, Blaze CA, Tucker HA. 1988. Effect of pinealectomy on prolactin, testosterone and luteinizing hormone concentration in plasma of bull caves exposed to 8 or 16 hours of light per day. J Anim Sci 66: 464-469.

24. Underwood H, Gross G. 1982. Vertebrate circadian rhythms: retinal and extraretinal photoreception. Experientia 38: 1013-1021.

25. Vale WG. 1988. Bubalinos: fisiologia e patologia da reprodução, Publ. Fundação Cargill, São Paulo, Brasil, 86 p.

26. Vale WG. 1994. Reproductive management of water buffalo under amazon conditions. Buffalo $J$ 2: 85-90.

27. Vieira F. 1985. Introdução à Bioestatística, $3^{\circ}$ ed., Ed. Campos, Rio de Janeiro, Brasil, 294 p.

28. Zicarelli L. 1999. Out-of-breeding-season mating technique in buffalo. In: Bubalinos: sanidade, reprodução e produção (Barnabe VH, Tonhati H, Baruselli PS ed.), Ed. Funep, Jaboticabal, Brasil, 202 p.

\section{SJR sclmaso SJR Rank}

\section{Powered by SCOPUS}

\section{Índice de impacto de Revista Veterinaria}

Noticias de Scimago Research Group (Scimago Journals \& Country Ranks, Scopus-Elsevier) comunican que la publicación de nuestra casa, Revista Veterinaria, continúa registrando índice de impacto. El índice SJR mide la influencia científica (impacto) del artículo de una revista, expresando cuán importante es el "artículo promedio" de la publicación en la discusión científica global (sistema Thomson Reuters).

Para nuestra revista, tal indicador había sido de 0,03 en 2008 y continuó en 2009, 2010 y 2011 exhibiendo ahora un nivel promedio de 0,05 (media de 3 últimos años). Asimismo, surge para nuestra publicación un sostenido descenso del indicador que relaciona "citas versus autocitas", demostrando que los autores de los artículos están abandonando la práctica de citar sus propias publicaciones anteriores.

Por último, se advierte que según este portal, nuestra "Revista Veterinaria" continúa siendo la única publicación de esta rama de la ciencia que posee índice de impacto en Argentina. Para el resto del cono sur tal distinción recae en Brasil (siete revistas), Chile (una revista), Colombia (una revista) y Venezuela (una revista). No registran índice de impacto las revistas de veterinaria de Bolivia, Paraguay, Uruguay, Perú y Ecuador.

Fuente:

http://www.scimagojr.com/journalrank.php?area $=3400 \&$ category $=0 \&$ country $=$ AR\&year $=2011 \&$ order $=$ sjr $\& \min =0 \&$ min_type $=\mathrm{cd}$ Retrieved June 4, 2012. 